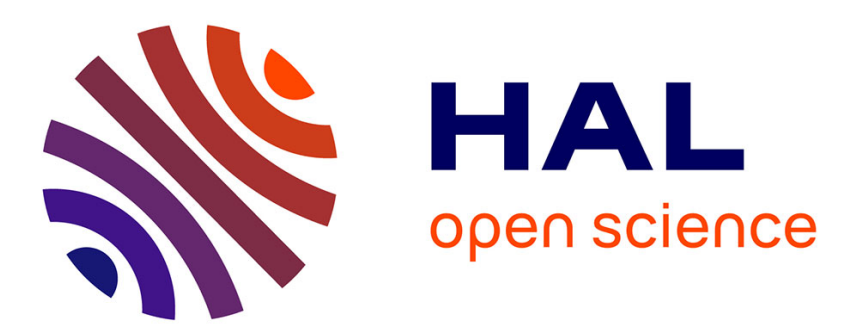

\title{
A method to determine the depowdered height in lattices manufactured by electron beam melting
}

Aurélien Carré, Matthieu Museau, Frédéric Vignat, Pierre-Thomas Doutre

\section{To cite this version:}

Aurélien Carré, Matthieu Museau, Frédéric Vignat, Pierre-Thomas Doutre. A method to determine the depowdered height in lattices manufactured by electron beam melting. Journal of Manufacturing Processes, 2018, 34, Part A, pp.1 - 6. 10.1016/j.jmapro.2018.05.025 . hal-01803858

\section{HAL Id: hal-01803858 https://hal.science/hal-01803858}

Submitted on 27 Feb 2019

HAL is a multi-disciplinary open access archive for the deposit and dissemination of scientific research documents, whether they are published or not. The documents may come from teaching and research institutions in France or abroad, or from public or private research centers.
L'archive ouverte pluridisciplinaire HAL, est destinée au dépôt et à la diffusion de documents scientifiques de niveau recherche, publiés ou non, émanant des établissements d'enseignement et de recherche français ou étrangers, des laboratoires publics ou privés. 


\title{
A method to determine the depowdered height in lattices manufactured by electron beam melting
}

\author{
Aurélien Carré $^{\mathrm{a}, \mathrm{b}, *}$, Matthieu Museau ${ }^{\mathrm{b}}$, Pierre-Thomas Doutre ${ }^{\mathrm{b}}$, Frédéric Vignat $^{\mathrm{b}}$

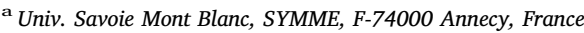 \\ ${ }^{\mathrm{b}}$ Univ. Grenoble Alpes, CNRS, Grenoble INP, G-SCOP, F-38000 Grenoble, France
}

\section{A R T I C L E I N F O}

\section{Keywords:}

Electron beam melting

Lattice structures

Depowdering

Powder recovery system

\begin{abstract}
A B S T R A C T
After completing a part in electron beam melting (EBM), a depowdering operation is required to separate the sintered but unmelted powder from the manufactured part. Depowdering lattice structures can be difficult or even impossible due to their intrinsic shape. The aim of this paper is to propose a criterion to ensure that a lattice structure manufactured by EBM can be depowdered. The objective is to use this criterion during the design phase of lattice structures to make them manufacturable and depowderable. Experiments are conducted on depowdering octet-truss lattice structures with variable bars thickness and mesh sizes. Different criteria are introduced, among them the criterion "hydraulic diameter" of a lattice structure, inspired by the Darcy-Weisbach hydraulic law used to calculate the pressure drop in a pipe. This criterion can be determined using only geometrical characteristics of lattices available in the CAD model of the part. Results show that the levels of depowdering for lattice structures are proportional to this hydraulic diameter. As a validation, a bike stem has been manufactured following the criterion and has demonstrated its efficiency.
\end{abstract}

\section{Introduction}

Nowadays, with the emergence of additive manufacturing processes [1], complex shapes that respondent at precise functional criteria can be produced [2]. We therefore want to push the limits in terms of design and optimization of parts, seeking to obtain the best possible shapes [3-6]. One way to achieve this goal is to use lattice structures that make possible to manufacture products lighter in weight with acceptable mechanical properties [7].

Electron beam melting (EBM) is an additive manufacturing process used for producing metal parts and has the potential to manufacture lattice structures with fine features [8]. EBM is a "powder bed" additive manufacturing process: the part is built layer-by-layer by melting the metal powder using a powerful electron beam $[9,10]$. For each layer, the metal powder is spread on a plate by a rake, is sintered by the electron beam to increase thermal and electrical conductivity and is locally melted to produce the required part. Then the plate goes down of the value of a layer (50) and the cycle is repeated (Fig. 1). During this study, the manufacturing has been done with an Arcam A1 machine [10]. The diameters of the titanium powder that we use range from 45 to $110 \mu \mathrm{m}$ and follow a distribution with a median value at $74 \mu \mathrm{m}$, with $d(0.1): 53.2 \mu \mathrm{m}$ and $d(0.9): 102.9 \mu \mathrm{m}$ [20].

At the end of the EBM process, the part is included in a "cake" of sintered powder. This powder has to be removed to obtain the final part and is then recycled. To perform this depowdering operation, a powder recovery system (PRS) [11] is used: the "cake" of sintered powder containing the manufactured part is blasted with titanium powder under 5 bar air pressure [12]. The removed powder is then sieved, so it can be reused. Depowdering lattice structures can be difficult or even impossible due to their intrinsic shape. The aim of this paper is to propose a criterion to ensure that a lattice structure manufactured by EBM can be entirely depowdered. The objective is to use this criterion during the design phase of lattice structures to design manufacturable and depowderable parts.

\section{Preliminary data and methods}

\subsection{Presentation of the structures}

The parts that are studied here are those with lattice structure. There are various geometries of lattice structures (diamond, octet-truss, dodecahedron, ...) with different properties $[13,14]$. Our study will deal particularly with octet-truss: this type of lattice is the best compromise in terms of mechanical properties [15].

The lattice structures are made by a unit cell that is repeated in the three directions (Fig. 2). Three parameters define this cell: the mesh size

\footnotetext{
* Corresponding author.

E-mail address: aurelien.carre1@univ-smb.fr (A. Carré).
} 


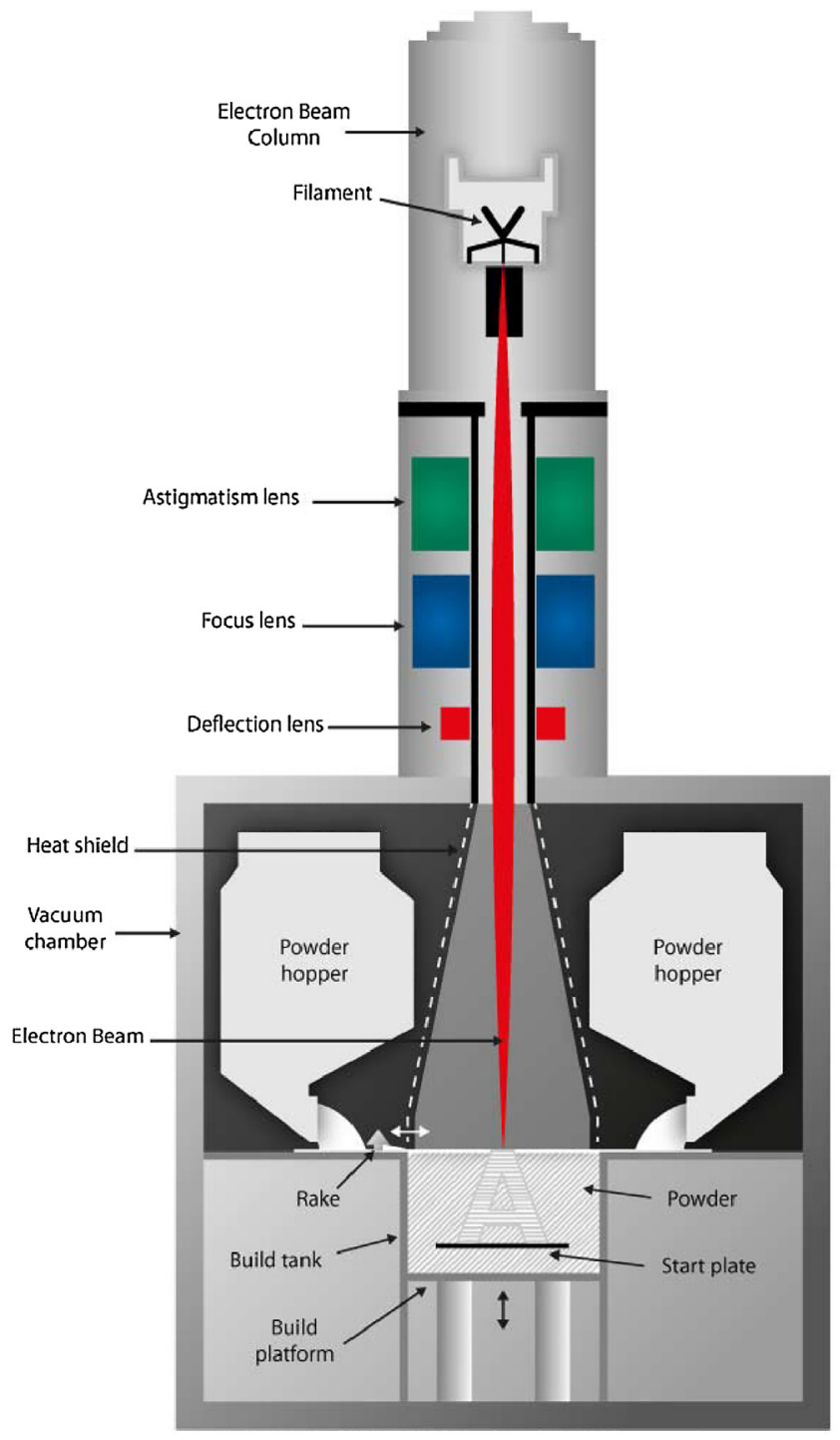

Fig. 1. Architecture of an EBM machine [10].

$p$, the bars thickness $e$, and the density of the lattice structure $d$ in percentage. One parameter is given by the value of the two others, so only two of them must be chosen.

The CAD is realized thanks to a macro that creates a cell with fixed $p$ and $e$, and then this cell is repeated in the three directions. The density $d$ in percentage is the ratio between the structure volume given by the CAD software and the full cube volume with the same external dimensions of the lattice structure. The manufacturing is prepared with Magics software [17] that allows to place and to orientate the parts on the support plate, and to take into account correcting coefficients due to thermal constraints during manufacturing (the geometry is not affected by any electrical effects).

The hypotheses taken and the boundaries of this study are:

- The structure density must be the same than the cell density; so the number of repetitions of the cell in the three directions must be an integer.

- The standard parameters of the EBM machine are used for manufacturing the part.

- Depowdering is studied only in one direction: the removed powder has to go out of the structure through the depowdered face. In that goal, the parts will have a "skin" on five faces, and the last one will be open (Fig. 2, right). This makes the worst case, because in a real case with an open lattice, the powder will go out through all faces, and the depowdering would be easier.

- The PRS system is used to depowder the parts, with a pressure of 5 bar, which is the maximum allowable value to allow optimal depowdering. The depowdering is done during $3 \mathrm{~min}$ : some studies show that it permits to remove more than $90 \%$ of the consolidated powder [12].

The structures studied can be divided in three groups: the first is composed of 8 unit structures (the unit cell is only repeated in the height), and 12 lattice structures (the unit cell is repeated in the three directions) with mesh sizes between $4 \mathrm{~mm}$ and $15 \mathrm{~mm}$. Those 20 parts have a density of $25 \%$ : this value is the best compromise between a light structure and a good rigidity, and allows a large choice of mesh size and bars thickness. The widths of the lattice structures are 24, 30 and $36 \mathrm{~mm}$, and the heights of the 20 parts are from $48 \mathrm{~mm}$ to $63 \mathrm{~mm}$, to satisfy the hypothesis of an integer number of repetitions.

The second group is composed of 8 square cylinders, with a width between $4 \mathrm{~mm}$ and $15 \mathrm{~mm}$, and a height of $49 \mathrm{~mm}$ (for a comparison with available data on depowdering circular cylinders $[12,16])$.

In the third group, there are 22 lattice structures with a density between $3 \%$ and $66 \%$ and mesh sizes between $4 \mathrm{~mm}$ and $12 \mathrm{~mm}$. The width and the height are 24 and $30 \mathrm{~mm}$. Those parts will permit to have a larger range of results.

\subsection{Calculation of the depowdered height}

Each part is weighed before and after depowdering: so the initial mass $m_{i}$ and the final mass $m_{f}$ are known.

For the square cylinders, the depowdered height is calculated as follows, with $S$ the hollow section and $\rho_{c}$ the density of the consolidated powder:

$h_{d}=\frac{m_{i}-m_{f}}{S \cdot \rho_{c}}$

For the lattices, the structure is more complex. In particular, the $1 \mathrm{~mm}$ thick skin is extended around the open face, to create a step that permits not to begin the lattice on the start plate (Fig. 3).

For calculations, $h_{t}$ is the lattice height, $S$ the lattice section, $V_{p, i}$ the initial volume of consolidated powder in the whole structure
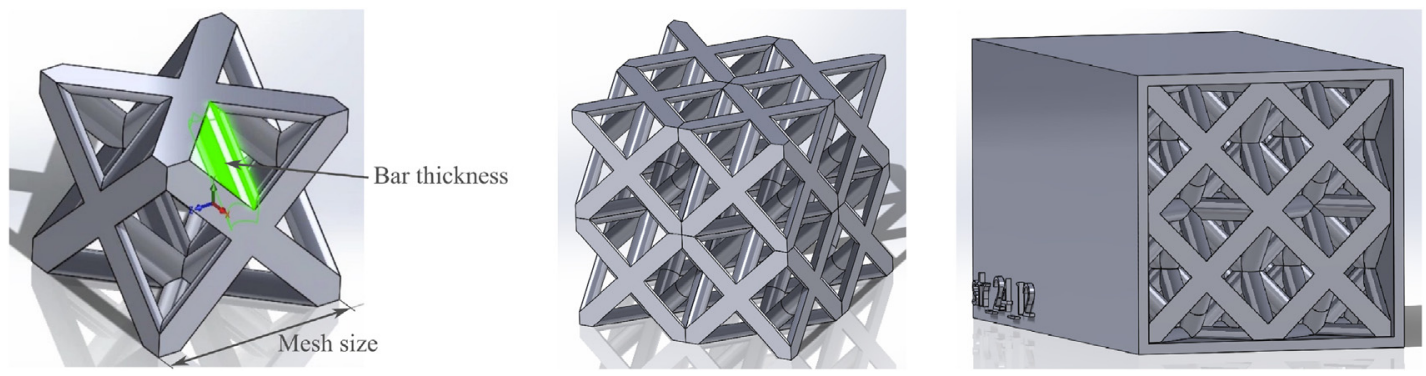

Fig. 2. Unit cell (left) and lattice structure. 


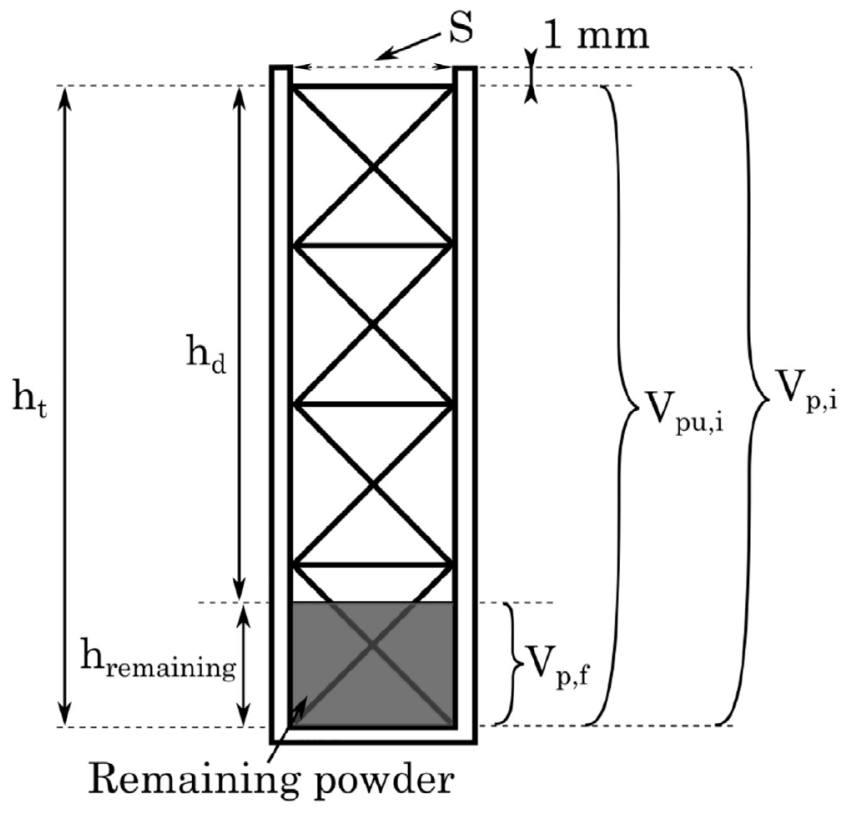

Fig. 3. Data for calculations.

(determined by CAD) and $V_{p u, i}$ the useful initial volume of consolidated powder (the volume taken by the melted lattice structure is not included in $V_{p, i}$ and $\left.V_{p u, i}\right)$. It is determined with:

$V_{p, i}=S \times 1+V_{\mathrm{pu}, i}$

The final volume of powder after depowdering is $V_{p, f}$. Thereby:

$$
\begin{aligned}
\frac{m_{i}-m_{f}}{\rho_{c}} & =V_{p, i}-V_{p, f} \\
& =V_{\mathrm{pu}, i}-V_{p, f}+S \times 1
\end{aligned}
$$

The depowdered height $h_{d}$ is calculated as follows:

$\frac{h_{d}}{h_{t}}=\frac{h_{t}-h_{\text {remaining }}}{h_{t}}=\frac{V_{\mathrm{pu}, i}-V_{p, f}}{V_{\mathrm{pu}, i}}$

So, with Eq. (3), it can be determined:

$h_{d}=\frac{h_{t}}{V_{\mathrm{pu}, i}} \cdot\left(V_{\mathrm{pu}, i}-V_{p, f}\right)=\frac{h_{t}}{V_{\mathrm{pu}, i}} \cdot\left(\frac{m_{i}-m_{f}}{\rho_{c}}-S \times 1\right)$

The calculated values are verified fore some parts, by dipping a thin iron wire and measuring the depth.

In order to predict the depowdered height in a structure, five different criteria are defined and then tested. Three of them are related to the free surface, and two of them to the hydraulic diameter. They are presented in the following of this paper.

\section{Free surface}

\subsection{Criteria}

The free surface $S_{l}$ in a structure is defined at different heights, as the portion of the surface without material (Fig. 4).

It can be determined thanks to a CAD software, because the occupied surface $S_{\text {occ }}$ (portion of the surface with material) is measurable: $S_{l}=(n p)^{2}-S_{o c c}$, with $p$ the mesh size and $n$ the number of repetitions of the cell in the plan. For a $24 \mathrm{~mm}$ width octet-truss structure with $p=12 \mathrm{~mm}$, the evolution of the free surface is represented in Fig. 5 .

The trend is the same for every octet-truss lattice, with a number of waves depending on the number of repetitions in the direction of depowdering.

From this point, it is possible to define three criteria that could be

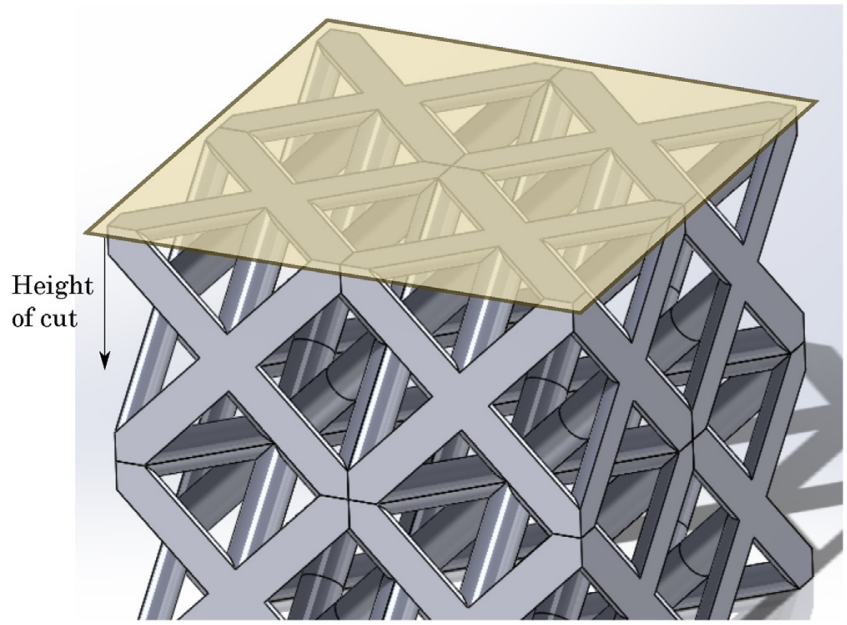

Fig. 4. Cut at different heights in the lattice.

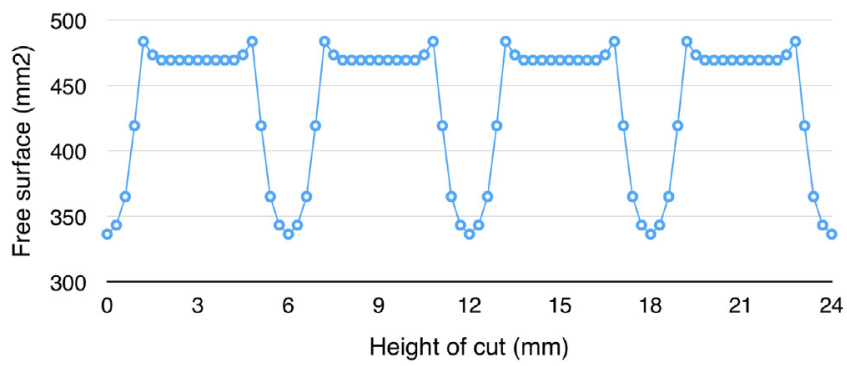

Fig. 5. Free surface function of the height of cut.

related to the depowdered height:

- The minimum free surface: $\min \left(S_{l}\right)$. In an octet-truss, it occurs in the open face, and can block the powder projection.

- The average free surface: $\operatorname{moy}\left(S_{l}\right)$. It cannot describe the sudden variations, but it represents the free surface in global.

- The gap between maximum and minimum of free surface: max $\left(S_{l}\right)-\min \left(S_{l}\right)$. It highlights the maximum variation in all the profile.

\subsection{Results}

Those criteria are calculated for all parts of the groups 1 and 2. The relation between the depowdered height and each criterion can be represented on a graphic. Only the graphic for the minimum of free surface is showed here, because for the other criteria it presents the same characteristics. The parts are divided in five colors, to better see the specificities of each type.

For the square cylinders and the unit structures, the criterion could be interesting because it reveals a linear relation with the depowdered height. Nevertheless, the most important results concern the lattice structures. For all external dimensions ( $24 \mathrm{~mm}, 30 \mathrm{~mm}$ and $36 \mathrm{~mm}$ ), the minimum of free surface is the same whereas mesh sizes are different, as it can be seen in yellow, orange and red in Fig. 6. For the two other criteria, the conclusion is the same: the criteria values are only linked to the lattice external dimensions, and thus are equal for different unit mesh sizes.

This highlights that these three criteria are not valid to predict the depowdered height: for example, if the value of the minimum of free surface is known, it is impossible to determine the height of powder that will be removed. With the same minimum of free surface, the depowdered height that is predicted can range from simple to triple. Finally, the criteria based only on the free surface cannot be used to determine the powder that could be depowdered on a random lattice 




Fig. 6. Depowdered height function of the minimum of free surface. (For interpretation of the references to color in text, the reader is referred to the web version of the article.)

structure.

In the following part, other criteria are proposed to fulfill the initial objective.

\section{Hydraulic diameter}

\subsection{Criteria}

The depowdering stage can be seen as a head loss of titanium particles due to friction. Between the open face, where the particle velocity is due to the standard parameters of the PRS and the bottom of the lattice, where the particles are stopped, we make the assumption that the kinetic energy loss and thus the head loss are constant.

In a general case in hydraulic, the head loss $\Delta H$ in canals are calculated with the Darcy-Weisbach law [18]:

$\Delta H=f_{D} \frac{L}{D_{h}} \frac{v^{2}}{2 g}$

With $f_{D}$ the pressure drop coefficient, $L$ the canal length, $g$ the gravity acceleration, $v$ the average speed of the fluid, and $D_{h}$ the hydraulic diameter, given by:

$D_{h}=\frac{4 S_{m}}{P_{m}}$

With $S_{m}$ the wetted area and $P_{m}$ the wetted perimeter.

This theory is applied to lattice structures: the goal is not to valid the Darcy-Weisbach law for those structures, but to make an analogy to find a possible rule about depowdering. The depowdered height that is researched corresponds to the length $L$ that is renamed $h_{d}$. It can be determined with the previous formula (Eq. (7)):

$h_{d}=L=\Delta H \frac{1}{f_{D}} \frac{2 g}{v^{2}} D_{h}$
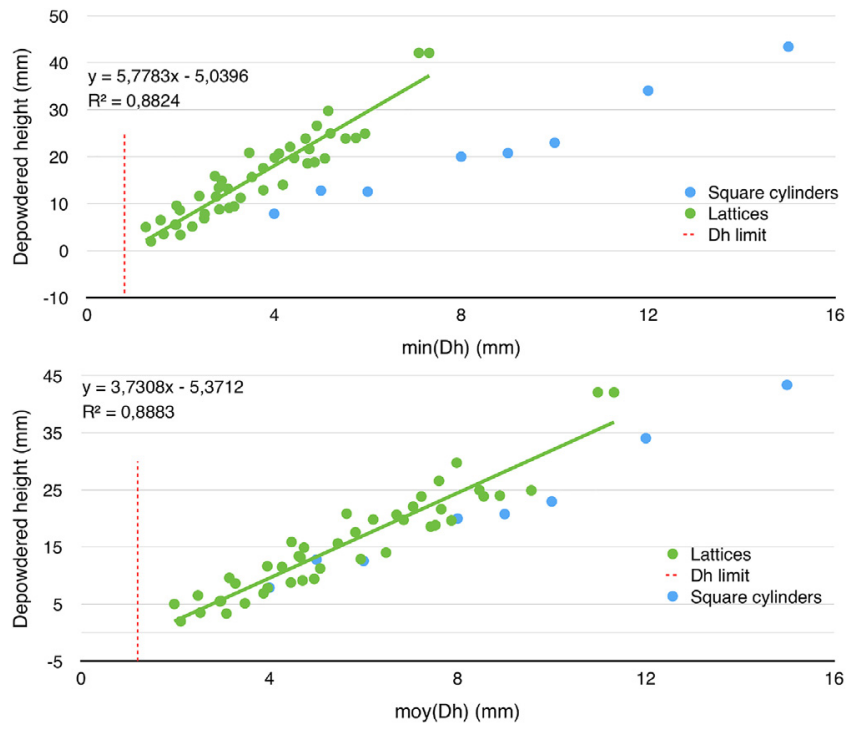

Fig. 8. Depowdered height function of minimum and average hydraulic diameter. (For interpretation of the references to color in text, the reader is referred to the web version of the article.)

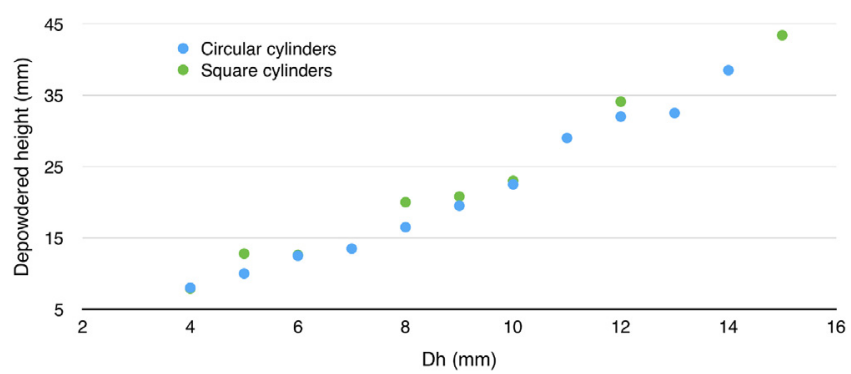

Fig. 9. Depowdered height function of hydraulic diameter.

depowdered height could be proportional to the hydraulic diameter: $h_{d}=K \cdot D_{h}$. If $D_{h}$ can be determined and $K$ is known, the value of $h_{d}$ can be specified.

As well as for the free surface, the hydraulic diameter is defined at every height of cut in the structure. The parameters needed are the wetted area $S_{m}$ and the wetted perimeter $P_{m}$. $S_{m}$ corresponds to the free surface $S_{l}$, so it is already known. For $P_{m}$, all the perimeters of the free surfaces are measured on CAD and are added (Fig. 7).

The minimum hydraulic diameter $\left(\min \left(D_{h}\right)\right)$ is obtained just on the open face of the lattice; it is the first criterion. Then, with the values of the hydraulic diameter at different heights of cut, it is possible to define another criterion, as it has been presented before for the free surface: the average hydraulic diameter $\left(\operatorname{moy}\left(D_{h}\right)\right)$.

The elements $\Delta H, f_{D}, g$ and $v$ are considered constant for the technology used and for the octet-truss lattice. Consequently, the
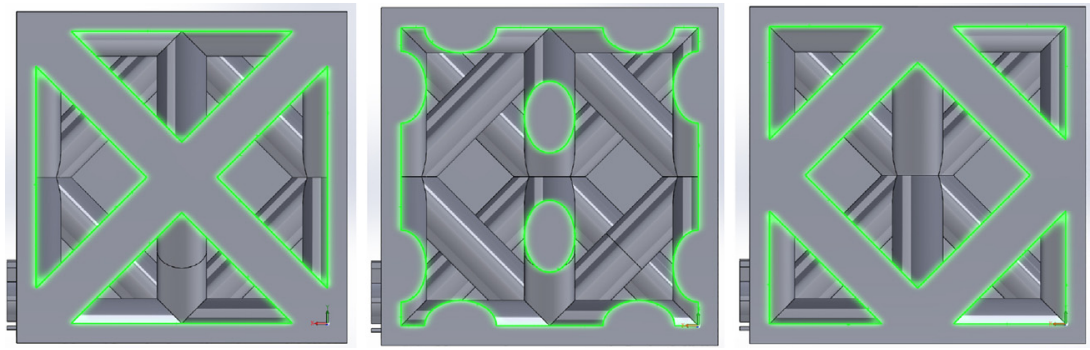

Fig. 7. Wetted perimeter (in green) for different heights of cut. (For interpretation of the references to color in this legend, the reader is referred to the web version of the article.) 

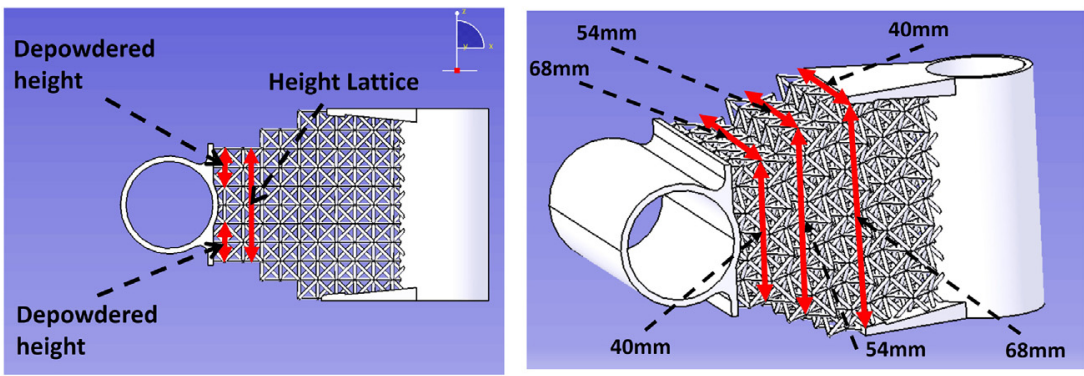

Fig. 10. Bike stem CAD with the lattice structure dimensions.

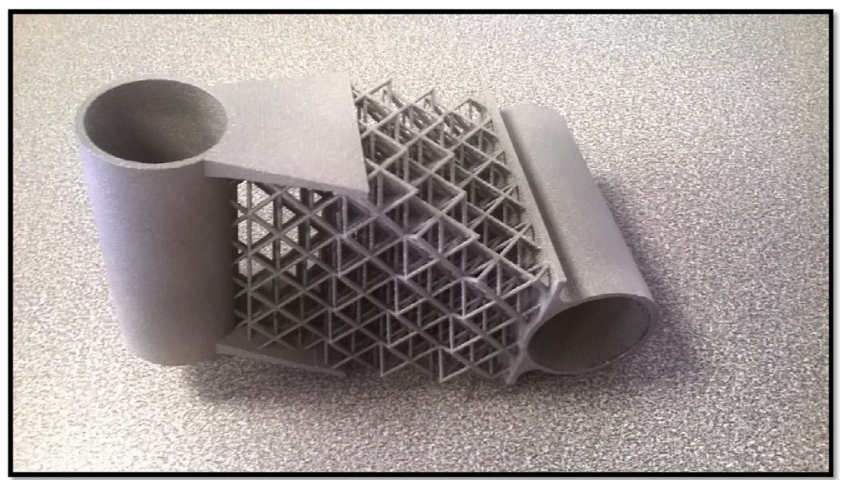

Fig. 11. Final bike stem after depowdering.

\subsection{Results}

For those two criteria, the parts of groups 1, 2 and 3 are used, to have results on a larger scale of data. Fig. 8 represents the depowdering height in a lattice function of the minimum hydraulic diameter and average hydraulic diameter. Square cylinders (group 2) are separated from the other parts, because they are not lattice structures: they are represented in blue and the lattices in green.

For the criterion "minimum hydraulic diameter", the depowdered heights of the lattice parts seem to follow a linear law: the vertical uncertainty is about $12 \mathrm{~mm}$ for the high values of $\min \left(D_{h}\right)$. The trend curve is represented, and its equation is precised. The leading coefficient (around 5.78) gives us directly the coefficient $K$ of the equation $h_{d}=K \cdot D_{h}$. Moreover, for an undepowdered part $\left(h_{d}=0 \mathrm{~mm}\right), \min \left(D_{h}\right)$ is not zero: it highlights its limit value (around $0.8 \mathrm{~mm}$ ), for which the powder would be totally blocked.

According to this criterion, square cylinders have different results than lattices, and do not follow the same law. But the relation between minimum hydraulic diameter and depowdered height seems also linear.

For the criterion "average hydraulic diameter", the same conclusions can be given: the depowdered heights of lattice structures follow a linear law. The limit of $\operatorname{moy}\left(D_{h}\right)$ for an undepowdered part is here of $1.2 \mathrm{~mm}$. For this criterion, square cylinders (in blue) almost follow the same law than lattices. This is interesting, because the use of a single criterion could give an estimate of the depowdered height, whether the structure is lattice or hollow.

It is also interesting to compare the square cylinders with the circular cylinders that have been studied $[12,16]$. For those shapes, the hydraulic diameter is constant in the whole part; it is simply the diameter for the circular cylinders. In Fig. 9 are represented the depowdered heights for the two kinds of cylinders.

It reveals that this criterion is also useful to predict the depowdering in cylinders: the two scatter plots seem to follow the same linear curve. With the use of the hydraulic diameter, it is possible to know in advance the depowdered height of cylinders, whatever their base geometry.

Finally, the criteria linked to the hydraulic diameter $D_{h}$ are very useful and valid to approximate the depowdered height of a structure (lattice or hollow) with its CAD model.

\section{Case study: lattice bike stem}

The criterion based on the minimum hydraulic diameter value presented in this paper has been used during the design phase of a product, to validate the possibility to depowder it. The product is a mountain bike stem: it has been designed with some octet-truss lattice structures. The design methodology detailed in [19] suggests, for this part and for an octet-truss pattern, a couple of parameters as follows: $e=0.63 \mathrm{~mm}$ and $p=13.6 \mathrm{~mm}$. This permits to create the CAD and to determine the minimum hydraulic diameter value: $\min \left(D_{h}\right)=4.9 \mathrm{~mm}$.

Thanks to the linear relation shown in Fig. 8, the expected depowdered height for this product is between 20 and $30 \mathrm{~mm}$. To ensure full part depowdering, no zone in the lattice structure should be at a distance from the external surface higher than the estimated depowder height. Considering the product geometry as shown in Fig. 10, no point is at more than $27 \mathrm{~mm}$ of the lattice bounding surfaces.

According to our criterion, depowdering the product could be feasible but is not guaranteed because the criterion is in the uncertainty zone. Nevertheless, decision is taken to produce the part.

The part has been manufactured with the same Arcam A1 machine, and the depowdering has been done with the PRS system using the same parameters than precised earlier. Finally, all the sintered powder in the bike stem is correctly removed (Fig. 11) and the proposed criterion for depowdering has been validated.

\section{Conclusion and prospects}

This study has given new tools for the design and the use of octettruss lattice structures in additive manufacturing, more precisely with the EBM technology. The depowder phase can now be understood more easily, and can be predicted. To do so, the use of two criteria, the average hydraulic diameter and the minimum hydraulic diameter, is possible. They are linearly linked to the depowdered height, and permit to approximate its value only with the CAD model. However, the value of the average hydraulic diameter is very long and tedious to calculate. It could be automated with a slicer, to obtain the value of the hydraulic diameter for each slice and then to determine the average. Though, the linear relationships that have been presented have only been validated for octet-truss lattice structures manufactured with the EBM technology, and depowdered with the PRS system. It would be interesting to apply this work to other types of lattices (diamond for instance), to have a more global view of the manufacturing possibilities. Finally, it could be useful to find another way to determine the value of $K$ coefficient for lattice structures, without need of experiences.

\section{References}

[1] Esmaeilian B, Behdad S, Wang B. The evolution and future of manufacturing: a review. J Manuf Syst 2016;39:79-100.

[2] Bourell DL, Beaman JJ, Leu MC, Rosen DW. A brief history of additive manufacturing and the 2009 roadmap for additive manufacturing: looking back and 
looking ahead. US-Turkey workshop on rapid technologies 2009:2.

[3] Saadlaoui Y, Milan JL, Rossi JM, Chabrand P. Topology optimization and additive manufacturing: comparison of conception methods using industrial codes. J Manuf Syst 2017;43:178-86.

[4] Shah P, Racasan R, Bills P. Comparison of different additive manufacturing methods using computed tomography. Case studies in nondestructive testing and evaluation vol. 6. 2016. p. 69-78.

[5] Salonitis K. Design for additive manufacturing based on the axiomatic design method. Int J Adv Manuf Technol 2016;87:989-96.

[6] Primo T, Calabrese M, Del Prete A, et al. Additive manufacturing integration with topology optimization methodology for innovative product design. Int J Adv Manuf Technol 2017:1-13.

[7] Suard M. Characterization and optimization of lattice structures made by electron beam melting [PhD thesis]. France: Univ. Grenoble Alpes; 2015.

[8] Vayre B, Vignat F, Villeneuve F. Metallic additive manufacturing: state-of-the-art review and prospects. Mech Ind 2012;13:89-96.

[9] Diegel O, Singamneni S, Reay S, Withell A. Tools for sustainable product design: additive manufacturing. J Sustain Dev 2010;3:68-75

[10] http://www.arcam.com/technology/electron-beam-melting/.

[11] http://www.arcam.com/technology/products/powder-handling/.
[12] Vayre B. Conception pour la fabrication additive, application à la technologie EBM [PhD thesis]. France: Univ. Grenoble Alpes; 2014.

[13] Hussein A, Hao L, Yan C, Everson R, Young P. Advanced lattice support structure for metal additive manufacturing. J Mater Process Technol 2013;213(7):1019-26.

[14] Yan C, Hao L, Hussein A, Raymont D. Evaluations of cellular lattice structures manufactured using selective laser melting. Int J Mach Tools Manuf 2012;62:32-8.

[15] Suard M, Martin G, Lhuissier P, Dendievel R, Vignat F, Blandin JJ, Villeneuve F. Mechanical equivalent diameter of single struts for the stiffness prediction of lattice structures produced by electron beam melting. Addit Manuf 2015;8:124-31.

[16] Vayre B, Vignat F, Villeneuve F. Identification on some design key parameters for additive manufacturing: application on electron beam melting. Proc CIRP 2013;7:264-9.

[17] http://software.materialise.com/magics20.

[18] Paraschivoiu I, Prud'homme M, Robillard L, Vasseur P. Mécanique des fluides. Presses internationales Polytechnique de Montréal; 2003.

[19] Doutre PT. Comment intégrer et faire émerger des structures architecturées dan l'optimisation de piéces pour la fabrication additive par faisceaux d'électrons $[\mathrm{PhD}$ thesis]. France: Univ. Grenoble Alpes; 2018.

[20] Petrovic Filipovic V, Niñerola R. Powder recyclability in electron beam melting for aeronautical use. Aircr Eng Aerosp Technol 2015;87(2):147-55. 